\title{
Muscle Activation of Soleus and Tibialis Anterior according to Landing Strategy of Initial Contact during Descending Stairs: Comparison of Forefoot and Whole Foot
}

\author{
Suwoong Choia, Yunbin Lee ${ }^{a}$, Taeyang Parka, Sujin Hwang ${ }^{a}$

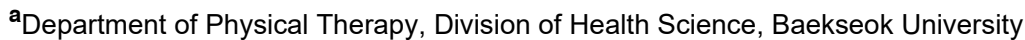

Objective: To control the rate at which body weight drops, forefoot initiates floor contact with the limb relatively extended at each joint. However, when the knee joints could not extend enough with going down the stairs, the forefoot cannot be contact initially. The purpose of this study was to investigate the differences between forefoot and whole foot in initial contract on soleus and tibialis anterior for health young adults when descending stairs.

Design: A cross-sectional observational study design.

Methods: Fifteen healthy young adults participated in this study. To compare between forefoot and whole foot in initial contact when going down the stairs, this study measured muscle activation on soleus and tibialis anterior. This study used the paired t-test to analyze the collected data and compare the supporting conditions.

Results: After analyzing, the muscle activation of soleus was not statistically significant difference as $25.16 \%$ at forefoot initial contact and $24.37 \%$ at whole foot initial contact when descending stairs ( $>00.05$ ). However, the muscle activation of tibialis anterior muscle was significantly difference was $49.19 \%$ at forefoot contact and $71.55 \%$ at whole foot contact.

Conclusions: The results of this study was that the muscle activation of the tibialis anterior was a higher at whole foot contact than that at fore foot contact when descending stairs. This study suggests that the landing strategy of the initial contact is a beneficial effect at the forefoot contact to maintain the postural balance and the muscle performance effectively when descending stairs in individuals with healthy young adults.

Key Words: Postural balance, Soleus muscle, Stair negotiation, Anterior tibialis

\section{Introduction}

Stair negotiations are a challenging and common factor in walking performance encountered in everyday life [1]. A staircase is a series of horizontal platforms with a vertical off-set between adjacent platforms to facilitate walking up or down a hill or other tilted surface [2]. The distinction between stand and swing phases of the stair descending are similar to overground walking, but stair descending would need the more muscle performance depending on the height and slope of the stairs compared to overground walking [3]. Stair negotiations require more capabilities of the magnitude of joint motion, the intensity of muscle action, and the forces generated during the ascent or descent than walking on flat overground [4]. Due to the nature of the stairs, it moves vertically and horizontally, and requires more muscle strength in the lower extremities compared to walking on flat ground to maintain Postural balance [5]. Motions on stair-like surface would change the muscle activations of the shine and calf muscles such as soleus and tibialis anterior (TA) muscles [6].

Received: Feb 15, 2021 Revised: Mar 4, 2021 Accepted: Mar 11, 2021

Corresponding author: Sujin Hwang (ORCID https://orcid.org/0000-0001-8471-0103)

Department of Physical Therapy, Division of Health Science, Baekseok University, P.O. Box 76, Munam-ro, Dongnam-gu, Cheonan-si, ChungcheongNam-do Republic of Korea, 31065

Tel: (+82)-41-550-2309 Fax: (+82)-41-550-2829 E-mail: sujin928@gmail.com

This is an Open-Access article distributed under the terms of the Creative Commons Attribution Non-Commercial License (http://creativecommons.org/licenses/ by-nc/4.0) which permits unrestricted non-commercial use, distribution, and reproduction in any medium, provided the original work is properly cited.

Copyright @ 2021 Korean Academy of Physical Therapy Rehabilitation Science 
The TA muscle is an essential element in the ankle and knee joints, plays an important role in bending the dorsiflexion of the foot and helps to keep constantly the axis of the ankle joint [7]. The TA muscle also needs to walk, run, and perform daily activities, and is unconditionally necessary for sports activities. The TA muscle weakness affects as a risk factor that may reduce postural stability while standing or walking. Weakness of the TA is deeply related to the limitations of walking speed, walking on stairs, and functional activities, and should be continuously managed and evaluated to maintain the movement and balance of the ankle joint [7]. Soleus as an antagonist muscle of the TA performs the plantarflexion together with the gastrocnemius muscles, generates about $93 \%$ of the torque of the plantarflexion, and plays a role in squatting or erecting the back $[8,9]$. It also plays an important role in posture control, and brings a sense of postural stability in walking speed than many types of plantar flexors. In walking, the EMG signal of the soleus muscle is the largest when moving the weight protrusion and when toe-off in gait cycle [1]. TA and soleus play a key role in maintaining postural balance and the TA muscle provides more proprioception than the soleus muscle $[6,10]$.

When maintaining balance, it is mainly controlled through the soleus and TA muscles. As the difficulty of postural control increases, the activations of these muscles increase [11,12]. Initial contact of gait cycle was made to the whole foot, not the forefoot when developing muscle fatigue of the TA muscle [13]. Previous studies have reported that the characteristics of worn shoes on muscle activations of soleus and TA [14]. During descending the stairs, the dominant functional task is to control the rate at which body weight drops. It is placed on the knee joints as the great demand, while ankle serves as the fulcrum for modification of limb alignment on the stairs [15]. There are still insufficient that studies on muscle activation of dorsiflexors and plantar flexors of the ankle joints according to the initial contact site of foot while descending the stairs [13-15]. This study hypothesized that there would be a difference in the muscle activation of the soleus and TA muscles depending on the initial contact area of foot when descending the stairs, because of generated power and muscle fatigue-accumulated. The purpose of this study was to investigate the differences between forefoot and whole foot in different initial contract on soleus and TA for health young adults when descending the stairs.

\section{Methods}

\section{Study participants}

Fifteen healthy young adults participated in this study. The inclusion criteria were as follows: a health young adults (1) without any orthopedic diseases to affect the results of this study, (2) without any neurological diseases, (3) who did not have orthopedic or neurosurgery damage within the last 1 year, (4) without any psychological problems, (5) who followed the instructions of the assessors, and (6) who spontaneously registered for participation. The experiment excluded all participants who did not continue to participate until the end of the study and did not participate in similar experiments within the last 6 months (Figure 1).

\section{Study design and approvals}

This study was a cross-sectional, observational study, that was approved by the Baekseok University's Scientific Ethical Committee (approval No. BUIRB202010-HR-027 All researchers in this study received appropriate ethical and scientific education and training for human subject research, and took responsibility for research ethics education. This study was based on the Helsinki Declaration, personal identification information was excluded and data collection was conducted. All participants of the study voluntarily agreed to participate in the study after receiving a full explanation of the purpose of the study. Participants in the study were informed in advance that they may withdraw from the study at any time during the course of the study, and that there is no penalty for it.

\section{Procedure}

This experiment was conducted for about 3 weeks from November 3 to November 25, 2020. Prior to the experiment, a sufficient explanation of the experimental process was given, and then properly recognizing that the participant had a minimum risk. This study involved two initial contact conditions including forefoot and whole foot conditions during stair descending. To measure the muscle activation on soleus and tibialis 


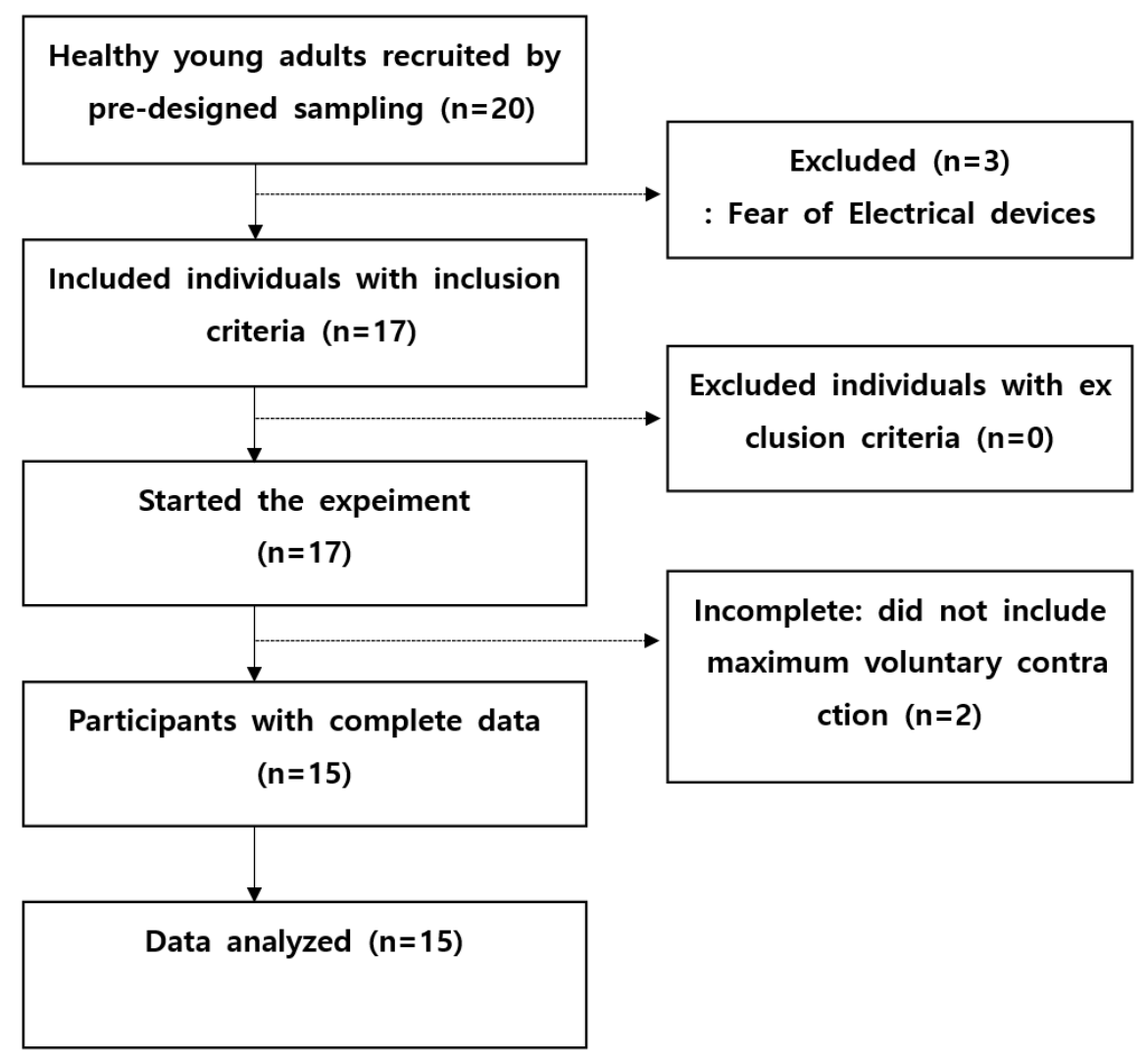

Figure 1. The flow diagram of this study

anterior, the participants selected the examination orders among A (forefoot condition and then whole foot condition), and B (whole foot condition and forefoot condition).

A ten-step stairs (step height $18 \mathrm{~cm}$, tread depth 28 $\mathrm{cm}$, and step width $120 \mathrm{~cm}$, handrails on both sides) was
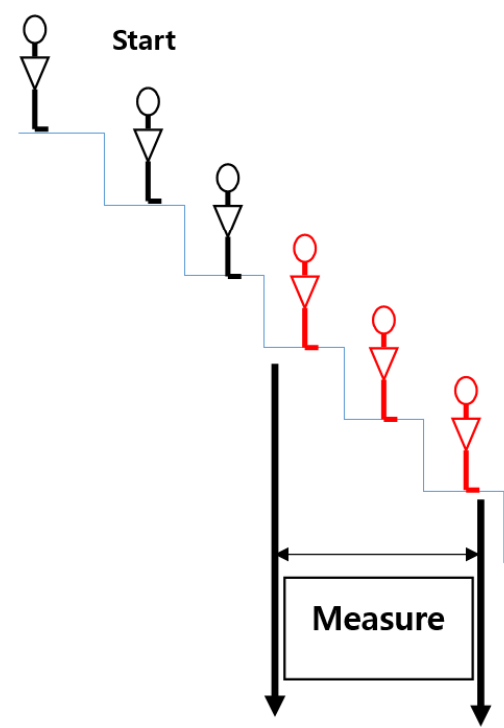

Condition \#1

: Forefoot co

ntact

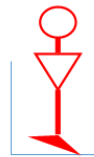

Condition \#2: Wh

ole foot contact<smiles>O=CCCCCCCCO</smiles>

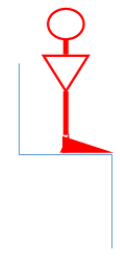

Figure 2. Experimental procedure of this study 
used to perform stair descent and a $200 \mathrm{~cm}$ walkway was extended at each end of the stairs. Participants performed level walking on the stairs followed by stair descent in a step-over-step manner and continued their walk for ten-step after stepping down from the stairs. All participants descended the stairs 20 times at a self-selected walking speed wearing their exercise shoes. An initial familiarization session with the staircase was allowed. Participants rested for $5 \mathrm{~min}$ after every five trials to exclude fatigue effects. The start points were spaced $50 \mathrm{~cm}$ apart to prevent memorization of the distance from the stairs. Two initial contact conditions were tested (1) forefoot contact and (2) whole foot contact. When the participant descended 10-step, 4-step in beginning and ending section were cut off and muscle activation was measured at 3-step in the middle session. Initial contact during the $200 \mathrm{~ms}$ on initial contact were determined via an electromyography to quantify muscle activity. Participant rested every 5 minutes and the average value of 3 measurements was used control the measurement error (Figure 2).

\section{Experimental equipment}

This experiment used the surface electromyography system (Trigno wireless biofeedback system; Delsys Inc. Co., USA) for measuring muscle activations of soleus and tibialis anterior muscles. The converted digital signal was processed using Trigno on a personal computer system. Signal sampling rate was set as 1,024 $\mathrm{Hz}$, and band pass filter of $20-500 \mathrm{~Hz}$ was used to minimize the noise. The collected signal was processed to root-mean-square. The surface electrodes were placed at $1 / 3$ on the line between the tip of the fibula and the tip of the medial malleolus for measuring the muscle activation of tibialis anterior muscle, and at $2 / 3$ of the line between the medial condyle of the femur to the medial malleolus.

\section{Statistical analysis}

Data from initial contact to stair descent transition is being utilized for the purpose of this study. The independent variables of this study were forefoot contact and total foot contact, and the dependent variable was ankle joint muscle activity. The general characteristics of the study subjects were analyzed by descriptive statistics. A paired t-test was used for initial contact comparisons between the forefoot contact and the power plant contact. The statistical significance level $(\alpha)$ was set to 0.05 . For statistical processing, the statistical package SSPS ver25.0 for Windows (SPSS ver25.0; IBM Co, USA) was conducted.

\section{Results}

In common characteristics of the participants, the average age was 24, the average height was $170.33 \mathrm{~cm}$, and the average weight was $65.27 \mathrm{~kg}$ (Table 1). The muscle activation of soleus was not statistically significant difference as $25.16 \%$ at forefoot initial contact and $24.37 \%$ at whole foot initial contact when descending stairs. However, the muscle activation of TA muscle was significantly difference between forefoot and whole foot at initial contact during descending stairs $(\mathrm{p}<0.05)$. The muscle activation of the TA was higher at

Table 1. Common characteristics of the participants in this study $(n=15)$

\begin{tabular}{llll}
\hline Variables & Mean (SD)/frequency (percent) & Minimum & Maximum \\
\hline Sex (male/female) & $10(66.37) / 5(33.33)$ & & \\
Age $(\mathrm{yr})$ & $24.00(1.41)$ & 21 & 26 \\
Height $(\mathrm{cm})$ & $170.33(10.15)$ & 155 & 183 \\
Weight $(\mathrm{kg})$ & $65.27(14.16)$ & 40 & 85 \\
\hline
\end{tabular}

*mean(SD), mean(standard deviation)

Table 2. Muscle activations during the initial contact of stair descending in this study $(\mathrm{n}=15)$

\begin{tabular}{lllll}
\hline Muscle & \multicolumn{2}{l}{ Mean \pm SD } & t & p \\
\cline { 2 - 3 } & Fore foot & Whole foot & 0.212 & 0.835 \\
\hline Soleus & $25.16(6.74)$ & $24.37(15.54)$ & -6.503 & $<0.001$ \\
Tibialis anterior & $46.16(13.95)$ & $71.55(11.41)$ & -503 \\
\hline
\end{tabular}


whole foot initial contact than forefoot contact as was $49.19 \%$ at forefoot contact and $71.55 \%$ at whole foot contact (Table 2).

\section{Discussion}

This study compared the muscle activity of the TA and soleus muscles between forefoot and whole foot at the initial contact when descending the stairs for health young adults. The results of this study were that the TA muscle activation was higher in whole foot's initial contact condition than the initial contact on forefoot, but the soleus muscle activation was not significantly different between two initial contact conditions.

Oskouei et al. [15] have investigated the muscle activation of the leg muscles involved in ascending and descending stairs with different heights participated by eighteen females. The results showed no significant effects of the muscle activation of the two muscles according to stair height [15]. Gerstle et al. [16] studied the influence of landing strategy and step height on lower extremity muscle activity of uninjured individuals during transition step descent. They evaluated the participant walked along a level walkway, stepped down a single step on the leading leg's peroneals, TA and medial gastrocnemius before and after initial contact landed with the heel or forefoot, and continued walking. They reported all muscle activity increased with each step height increment during the post-contact period, so it is important the landing strategy and step height when designing or interpreting investigations of transition stair negotiation [16]. This study examined the muscle activations on soleus and TA muscle between two landing strategies of the leading limb, forefoot and whole foot's initial contact during descending stairs. This study showed the different muscle activation on the TA muscle, that are higher activities during whole foot landing condition compared with the forefoot landing condition.

This study paid attention to the previous studies that the landing strategy is very important in preventing falls when going down stairs. Over one quarter of the ankle sprains requiring hospital care over a four year period were the result of a fall from stairs and falls on stairs occur three times more often during descent than ascent. During the stairs descending, lower extremity musculature is critical to the positioning of the foot and ankle for initial contact and stabilizing the structures fallowing contact $[16,17]$. Therefore, an efficient muscle performance without fatigue involved in maintaining proper landing and postural stability can be seen as important for safe stair descending and prevention of falls. This study focused on to compare the landing strategy for descending stairs was to close the forefoot with the whole foot to improve the abilities to get tired or to improve postural balance. In addition, this study compared the muscle activities of soleus and TA, which are mutually antagonistic muscles among the external muscles that act upon the initial contact according to the landing strategy. Unlike previous studies that compared just before and right after landing of the leading limb, this study measures electromyography at the muscle activations of soleus and TA on initial contact [16]. Gerstle et al. [16] reported that the muscle activity of the plantar flexors was higher than that of walking with the power generation regardless of the height of the stairs when walking with the forefoot touched. However, this study was not different on the soleus muscle activation between forefoot and whole foot in initial contact when descending stairs, but was higher the TA muscle activation on whole foot at initial contact than that of forefoot at initial contact. This study suggests that the while foot landing at initial contact would be negative effects on muscle fatigue of TA muscle compared with forefoot landing strategy and is a risk factor for losing the postural balance during descending stairs.

This study examined the difference in muscle activations of soleus and TA according to different initial contact with descending stairs in health young adults, and showed the results which the TA muscle activation was higher in whole foot condition than in forefoot condition, but not soleus muscle activation. As such, it can be seen that choosing a foot strategy when descending stairs can have a great influence on the muscle activity of the TA muscle are affected. To control the rate at which body weight drops, forefoot initiates floor contact with the limb relatively extended at each joint. However, when the knee joints could not extend enough with going down the stairs, the forefoot cannot be contact initially. However, this study could not measure the knee joints muscles. In future studies, it will be necessary to compare the muscles around the knee joints when descending the stairs. This study participated 
the healthy young adults, so future studies involving osteoarthritis or elderly persons are required to examine the effect of landing strategy on muscle activations of ankle and knee joints during descending stairs. However, this study was a pilot study with a observational, cross-sectional study design. Future studies should examine the effectiveness of landing strategy on muscle activations of ankle and knee joints during descending stairs participated by large sample size.

\section{References}

1. Sheehan RC, Gottschall JS. Stair walking transitions are an anticipation of the next stride. J Electromyogr Kinesiol. 2011;21:533-41.

2. Perry J. Gait analysis. Thorofare, NJ: SLACK ;; 1992.

3. Kim DY, Park GI, Jang YW, Park SY. Kinematic and kinetic comparison between stair climbing and level waling. Ann Rehabil Med. 2001;25:1048-58.

4. Moon JH, Chun YJ. Biomechanical analysis of the Effect that various loads has on the lower limbs while descending stairs. Korean J Sport Biomechanics. 2013;23:245-52.

5. Nam K-J, Lee C-H. The difference of EMG between of Up-stair gait and Down. Korean J Sports Sci. 2017;26:1247-54.

6. Choi T, Im CH, Kim SJ, Kim H, Lee JM. Prediction Method of Walking Speed at Swing Phase using Soleus Electromyogram Signal at Previous Stance Phase. Annu Int Conf IEEE Eng Med Biol Soc. 2018;2018:2308-11.

7. Jeon I-C. Comparison of the Electromyographic Activity of the Tibialis Anterior and Isometric Dorsiflexor Strength during Dorsiflexion According to Toe Postures in Individuals with Ankle Dorsiflexor Weakness. J Kor Phys Ther. 2020;32:233-7.

8. Lee HS, Hong SB, Chin HN, Choi JL, Seon HC, Jeong DY. Characteristics of Muscle Activity in the Lower Extremity during Stepping over Various Obstacle. J Korean Soc Phys Med. 2019;14:55-62.

9. Schoenfeld BJ, Vigotsky AD, Grgic J, Haun C, Contreras B, Delcastillo K, et al. Do the anatomical and physiological properties of a muscle determine its adaptive response to different loading protocols? Physiol Rep. 2020;8:e14427.

10. Riemann BL, Myers JB, Lephart SM. Comparison of the ankle, knee, hip, and trunk corrective action shown during single-leg stance on firm, foam, and multiaxial surfaces. Arch Phys Med Rehabil. 2003;84:90-5.

11. SW H, YW P, JR Y, MJ K, YW C, HB J, et al. Electromyographic analysis of ankle muscles according to unstable platforms. J Coaching Develop. 2006;8:231-9.

12. Shin S, Kim M, Ko H, Jeong H, Hong C, Kwon TK. Analysis of Brain and Muscle Activity in Maintaining Postural Balance According to the Ground Conditions. Transactions of the Korean Society of Mechanical Engineers - B. 2020;44:169-75.

13. Garin Lim, Chang J. The effects of muscle fatigue of knee muscles on balance ability in healthy adults. J Korean Med Rehabil. 2018;28:105-11.

14. Shang J, Chen L, Zhang S, Zhang C, Huang J, Wang $\mathrm{X}$, et al. Influence of high-heeled shoe parameters on biomechanical performance of young female adults during stair ascent motion. Gait Posture. 2020;81: 159-65.

15. Eteraf Oskouei A, Ferdosrad N, Dianat I, Asghari Jafarabadi M, Nazari J. Electromyographic Activity of Soleus and Tibialis Anterior Muscles during Ascending and Descending Stairs of Different Heights. Health Promot Perspect. 2014;4:173-9.

16. Gerstle EE, Keenan KG, O'Connor K, Cobb SC. Lower extremity muscle activity during descent from varying step heights. J Electromyogr Kinesiol. 2018;42:57-65.

17. Waterman BR, Owens BD, Davey S, Zacchilli MA, Belmont PJ, Jr. The epidemiology of ankle sprains in the United States. J Bone Joint Surg Am. 2010; 92:2279-84. 